\title{
Energy Quality in Voltage, Current and Power Signals
}

\author{
T. Yebra, V. Fuster \\ Instituto de Tecnología Eléctrica \\ Parque tecnológico de Valencia \\ 46022 Valencia (Spain) \\ Phone/Fax number:+0034 961366670 / +0034 961366680 \\ e-mail: tomas.yebra@ite.es, vicente.fuster@ite.es.
}

\begin{abstract}
This article deals with the processing of electrical signals for use in the analysis of the quality of electrical energy in installations. The calculations are based on a three-phase wave, taking into account the evolution in time of the sequential components. The currents within the system are also factored in, and one of the diverse theories of power in non-sinusoidal regimes is applied.
\end{abstract}

As a result of the described processing, it was possible to show that the three-phase wave enables a better level of compression of the physical phenomena causing perturbations. Future studies will focus on quality parameters of electrical current signals, as these provide information regarding charge problems. The measurement of power brings a new focus, which allows the evaluation of perturbations according to their energy efficiency.

\section{Key words}

Signal processing, electrical perturbations, power in non-sinusoidal regimes, voltage dips.

\section{Introduction}

This study centres on the measurement of electrical perturbations. There are two parts to the project. The first centres on the processing of electrical signals, and the second applies one the theories of the measurement of electrical power in non-sinusoidal regimes. In order to test the validity of this approach, the algorithms studied were applied to measurements taken in power installations.

Using signal measurements recorded using registering devices, a study is performed to determine the appropriate algorithm to apply when dealing with signals in perturbed regimes. The results of this study indicate that the Short Time Fourier Transform (STFT) is an appropriate tool for the project. The STFT is used to process the voltage and current signals. In this way, the time sequence of the magnitudes and phases of each of the signals. From these calculations, the components of the direct, inverse and homopolar sequences for voltage and current are obtained by means of the StockvisFortescue Transform. This set of signals provides information about the severity of the transformation, the phase(s) where it was generated, the duration; the shape of the waves present can also give some idea about the cause of the perturbation.

As there is no consensus in the scientific community about the best one to use, various theories of power in non-sinusoidal regimes were studied, and the one best adapted to the project was evaluated. The theory of V. León was adapted for use with the STFT, and applied to transitory regimes. This enabled the measurement of apparent power, effective power, reactive power, distortion power and the efficiency factor. Examination of these power signals and their evolution allows us to differentiate between those perturbations which improve the efficiency of the installation and those which decrease it. In this manner it is possible to detect whether there has been a charge increase or decrease, whether harmonics have been introduced or eliminated, and also to quantify numerically the imbalance introduced by the perturbation.

It is safe to conclude that an approach where both voltage and current signals are taken into account provides valuable information for the managers of power station installations. On the other hand, an energy-based approach might well prove difficult, as the expressions in power theory are often complex. Quantitative information on aspects such as the asymmetry or distortion for the entire three-phases system are obtained; until now, this information could only be obtained for groups of signals (voltage or current), or a single signal in the case of distortion.

\section{Algorithms for non-sinusoidal regimes}

Signal processing is a key step when dealing with signals in transitory regimes such as electrical perturbations. It is necessary to arrive at a compromise between time and frequency resolution; according to the type of technique used, one may achieve more or less flexibility in each of these axes [1].

\section{A. Window and frequencies}

When the STFT is deployed to analyse a signals in a stationary regime for a period of time, there are just two possible strategies for obtaining good frequency 
resolution: either very rapid sampling over a short duration, or slower sampling over a much larger time period. A perturbation to be analysed is produced within a limited time interval; so if it is not very rapid, one may take the interval in which it is produced and analyse the signal as if it were stationary. If the perturbation is very rapid, this focus or approximation causes a loss of frequency resolution.

\section{B. Wavelets}

The wavelet transform (WT) [2] allows variation of the window size, in order to obtain a good frequency resolution in those intervals where major signal variations exist. Thus the WT utilises narrow windows for high frequencies, and wide windows at low frequencies. Figure 1 shows a representation of the divisions in the time-frequency plane, made during the analysis.

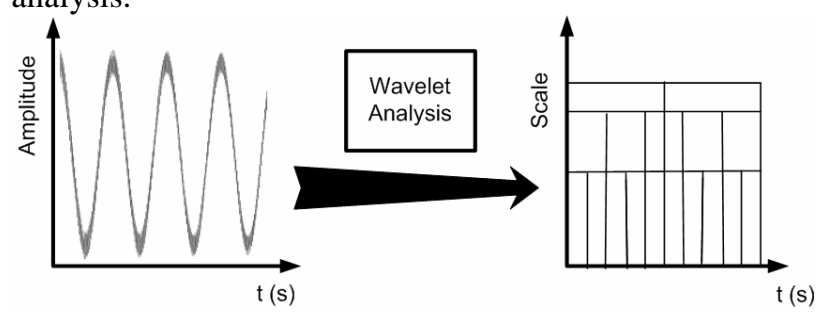

Fig. 1. WT in time-frequency plane

\section{Short Time Fourier Transform}

This method involves multiplying the signal by a sliding window of fixed size, and applying to it the FFT algorithm. These operations yield a time-frequency representation as shown in figure 2 .

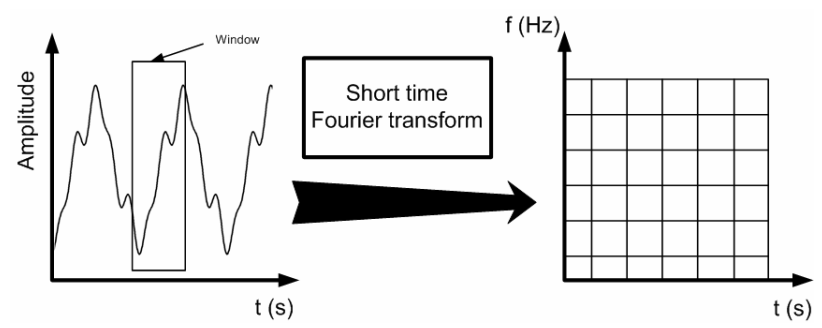

Fig. 2. STFT in time-frequency plane

The STFT presents problems in event detection if good frequency resolution is required. If the event is of very short duration, large errors emerge in the calculations of magnitude and angle. Errors in the angle calculation are also generated if the fundamental component frequency varies during the event.

Nevertheless, when the STFT is applied with a sliding window for each sample, good results are obtained for the analysis of the quality of supply.

This tool was selected for the analyses because:

1) it provides information about the magnitude and phase of the signal
2) while event detection is not as good as with the $\mathrm{WT}$, it is acceptable if the window is extended for each sample

3) it has better immunity to noise than the other techniques

4) it is rapid, enabling real-time applications

\section{Database of electrical registers}

The signals were processed for a large group of electrical registers. These registers were taken from substations and transformer centres in the Valencia Region. In addition, existing infrastructures, created for other studies [3], could be utilised here.

\section{A. Database structure}

The database was implemented using MySQL®. Figure 3 shows the tables comprising the database and its relational structures. The tables may be divided into two types: those storing data taken directly from the systems, and those storing results of processing.

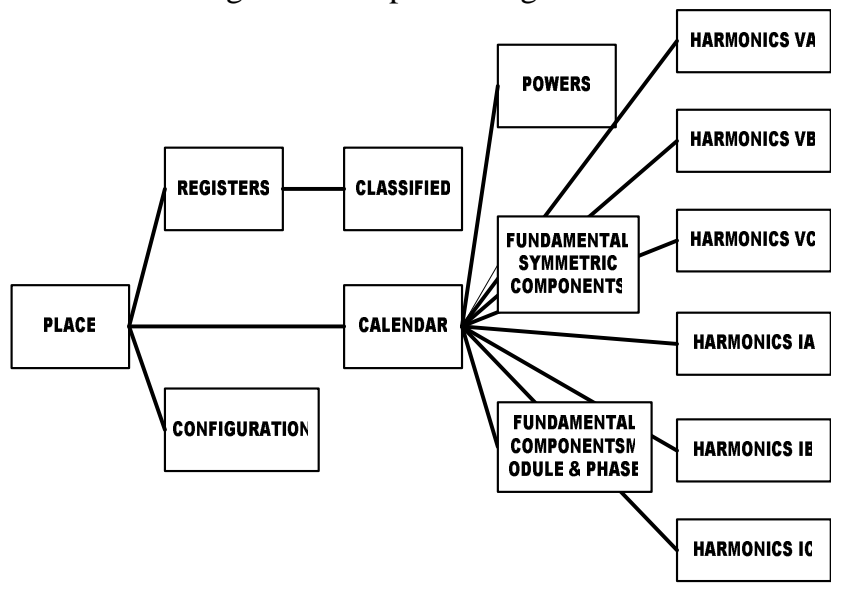

Fig. 3. Relationships among the tables in the database

In the first group are the following tables:

Place: Stores the names of the substations and power lines where measures have been taken.

Configuration: Stores the type of equipment used for the register and the current transformation ratios.

Registers: Stores simple voltages and currents, and voltages on neutral phase, as measured in the register.

Calendar: Stores the day and hour of the event.

Classified: Stores the classification of the event recorded by the register.

In the second group are stored the processing calculations performed on the registers. This enables rapid consultations, as the calculations are only performed once. The names of the tables indicate the class of data stored.

\section{B. Graphical interface}


Figure 4 shows the graphical interface developed using MATLAB to facilitate consultations of the database and provide functionality for register analysis. The functions implemented enable:

1) Register selection by substation, line measurement point, day and time.

2) Creation of variables in MATLAB workspace for the magnitudes measured.

3) Shows, in a window, the voltage and current measurements in terms of magnitude and angle for the 3 phases and neutral.

4) Shows the time development of the symmetrical fundamental components of currents, and the voltages, powers and efficiency.

5) Shows the spectrum of the harmonics at the instant selected, and the time development of the harmonic up to the 32 nd order.

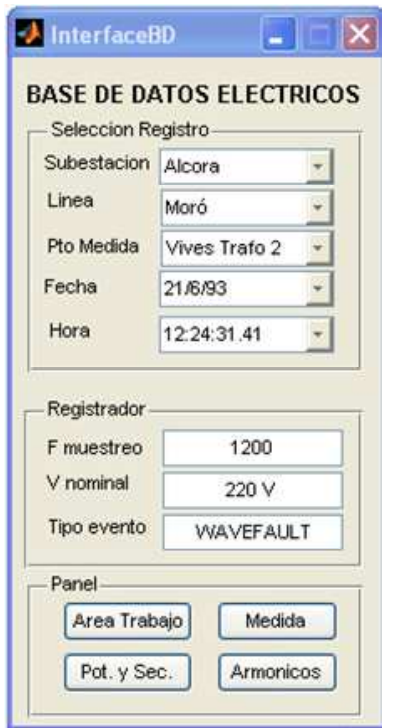

Fig. 4. Graphical interface for the manipulation of electrical registers stored in the database.

\section{Unified theory of electrical power}

Various authors have advanced theories in the area of electrical power in non-sinusoidal regimes which attempt to measure non-linear phenomena, when the voltage and current signals are unbalanced or deformed. There are two main schools of thought: the theorists who work in the frequency domain (Budeanu) [4], and those who follow a time-based approach (Fryze) [5].

There is no consensus within the scientific community regarding the best theory to use. However, the expressions developed by Budeanu have been recorded in the IEEE Standard Dictionary. On the other hand, the IEEE Power Engineering Society recommends the work of Kuster and Moore [6], which follows the approach of Fryze. These theories do not cover all topologies or all the non-linearities of electrical systems. In recent years the work of L.S. Czarnecki [7],[8] has had a significant impact, although this theory presents some problems, according to V. León [9].

\section{A. Formulation of the theory}

The theory of V. León [9],[10] was selected, as:

1) The components of current confirm Kirchoff's First Law around a closed circuit.

2) The expressions for instantaneous and apparent power have the same terms, i.e. they are mathematically similar.

3) All the components divided by the instantaneous and apparent power fulfil the superposition principle.

4) This theory encompasses all possible topologies in electrical systems. Specifically, we have, generator side: single-phase sinusoidal, single-phase non-sinusoidal, balanced three-phase sinusoidal, balanced three-phase non-sinusoidal and unbalanced three-phase nonsinusoidal. Load side, we have: linear single-phase, nonlinear single-phase, balanced linear three-phase, balanced non-linear three-phase, asymmetric linear three-phase and asymmetric non-linear three-phase.

From the definition of instantaneous power, according to equation 1 :

$$
p(t)=v(t) \cdot i(t)=\sum_{j} v_{j}(t) \cdot \sum_{k} i_{k}(t)
$$

The instantaneous power factors into various power terms, each characterising one of the non-linear energy phenomena.

$$
p(t)=p_{e}(t)+p_{r}(t)+p_{A}(t)+p_{D}(t)
$$

where

$\mathrm{p}_{\mathrm{e}}(\mathrm{t})$ is the effective instantaneous power,

$$
p_{e}(t)=\sum_{z} v_{a d 1 z} \cdot i_{a d 1 z}
$$

$\mathrm{p}_{\mathrm{r}}(\mathrm{t})$ is the instantaneous reactive power,

$$
p_{r}(t)=\sum_{z} v_{a d 1 z} \cdot i_{a d 1 z}
$$

$\mathrm{p}_{\mathrm{A}}(\mathrm{t})$ is the instantaneous power of asymmetry

$$
p_{A}(t)=\sum_{z}\left(\sum_{\substack{u=i, h \\ j, k=a, r}} v_{z j u n} \cdot i_{z k u 1}+\sum_{\substack{x \neq y \\ x, y=d, i, h \\ j, k=a, r}} v_{z j x 1} \cdot i_{z k y 1}\right)
$$

And $\mathrm{p}_{\mathrm{D}}(\mathrm{t})$ is the instantaneous distortion power.

$$
p_{D}(t)=\sum_{z}\left(\sum_{\substack{n=2 \\ x, y=d, i, h \\ j, k=a, r}} v_{z j x n} \cdot i_{z k y 1}+\sum_{\substack{n \neq m \\ x, y=d, i, h \\ j, k=a, r}} v_{z j x 1} \cdot i_{z k y 1}\right)
$$

The sub indices have the following significance: $\mathrm{z}$ is the phase number in a three-phase system, a is the active 
component, $r$ is the reactive component, $d$ the direct sequence component, $\mathrm{i}$ the inverse sequence component, $\mathrm{h}$ the homopolar sequence component, $\mathrm{n}$ is the order of the harmonic and 1 the fundamental harmonic.

\section{Application example}

In this section the signals obtained from the analysis of a voltage dip in one of the power installations are expounded. Figure 5 shows voltage and instantaneous current in the three phases of a fault measured on the medium voltage side.
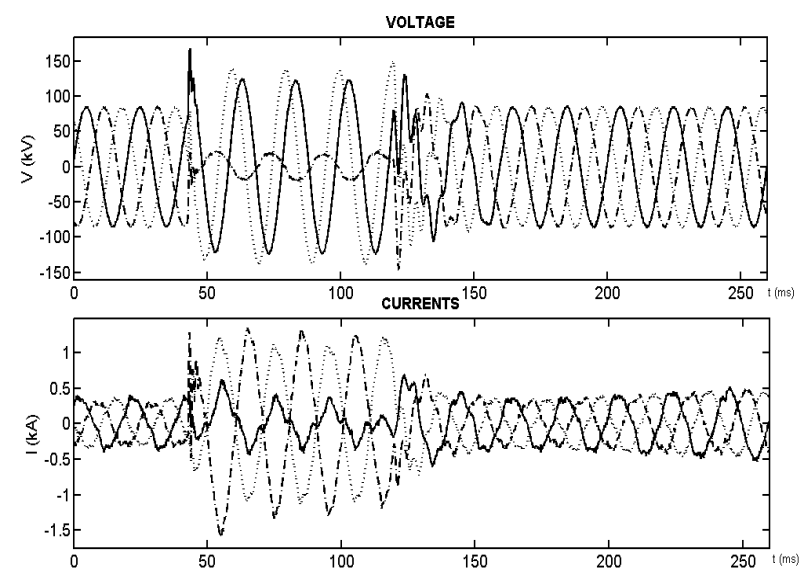

Fig 5. Recording of voltages and currents during a voltage dip

\section{A. Magnitude and angle values}

Once the perturbation has been registered, the STFT is applied to the currents in order to determine the time evolution of the magnitude and angle of the voltages and currents. The results are shown in figure 6 . Focusing on the effective voltage values, it may be concluded that the dip generated a single-phase fault due to the sharp fall in the phase $\mathrm{C}$ amplitude. Concurrently, the voltage in the other two phases increases, because there is generation capacity connected through the LV network.
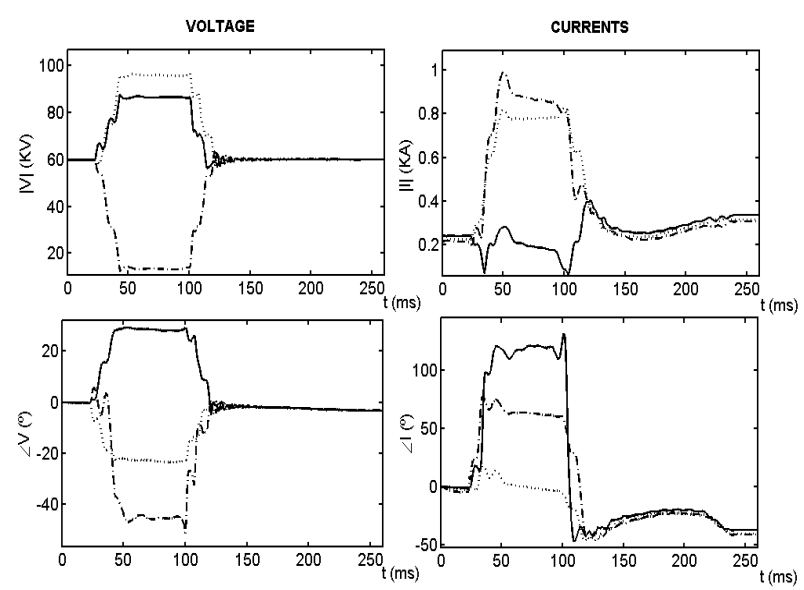

Fig. 6. Register of voltages and currents during a voltage dip.
The system also enables the calculation of the time evolution of the direct, inverse and homopolar sequences, as shown in figure 7 .

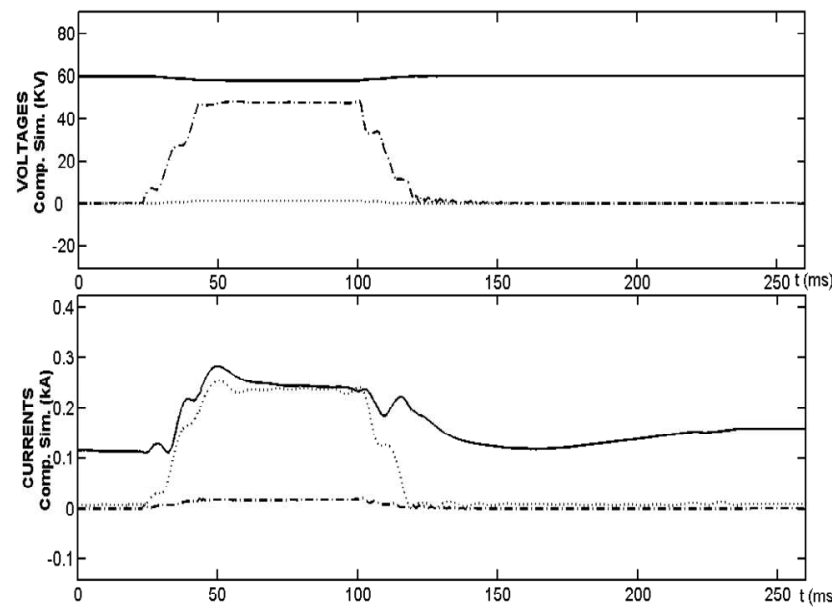

Fig. 7. Time evolution of voltage and current symmetric components. Legend: — Sec. Direct. ---- Sec. Inverse. -.-- Sec. Homopolar

\section{B. Harmonics}

Two formulations have been used to represents the harmonics. The first, shown in figures 8 and 9 , is based on the representation of the spectrum of the first 32 signal harmonics through a window of one cycle. Figure 8 shows the signal spectrum during the voltage dip. Figure 9 shows the spectrum during one of the transitory periods of recuperation from the dip.

The second representation shows the time evolution of the harmonic. In figure 10, the evolution of the third harmonic $(150 \mathrm{~Hz})$ of voltage for the three phases is represented.

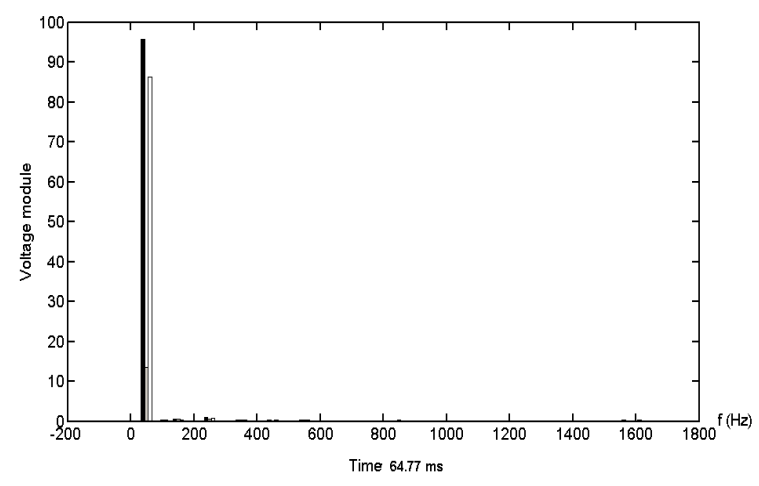

Fig. 8. Spectrum of voltages during the dip. The instant of initiation of the period is at $64.77 \mathrm{~ms}$.

The analysis tool enables the display of both types of representation, both for voltage and current, at any instant in time for any harmonic up to the 32 nd order. 


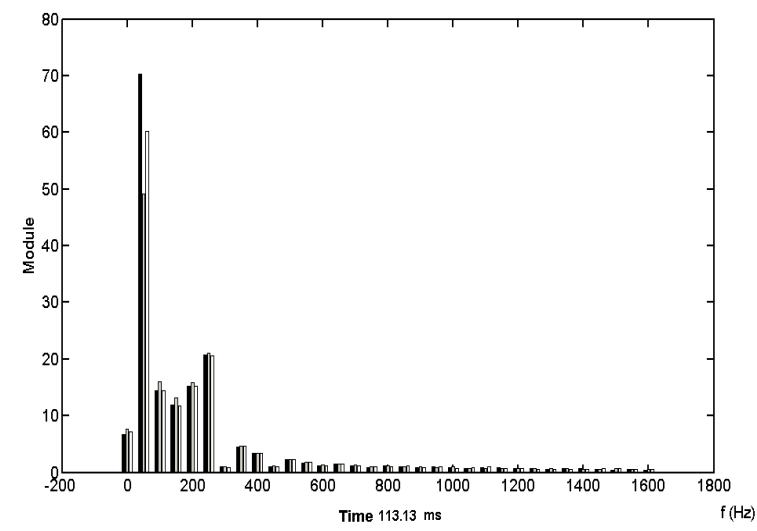

Fig. 9. Spectrum of voltages during the transient time of recuperation form the dip.
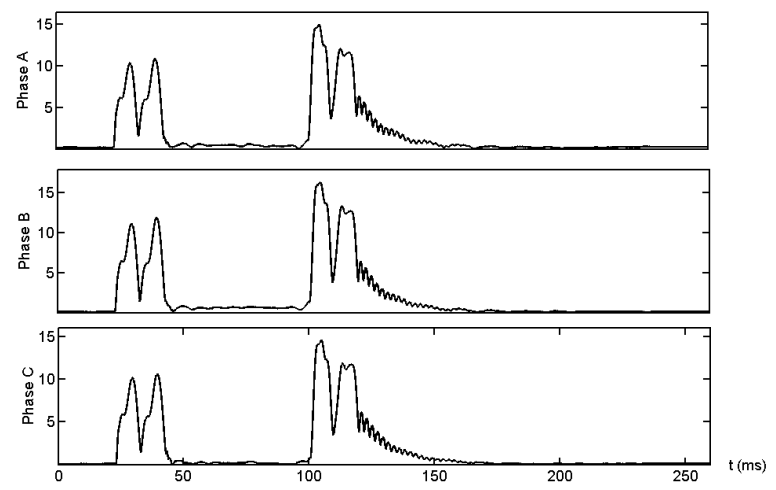

Fig. 10. Time evolution of the 3rd order voltage harmonic

\section{Power expressions}

Once the voltage and current phasors have been computed, the expressions from the unified theory of electrical power may be applied. Values are obtained with a window of one cycle updated for each sample.

Figure 11 shows the instantaneous power calculated according equation 1 .

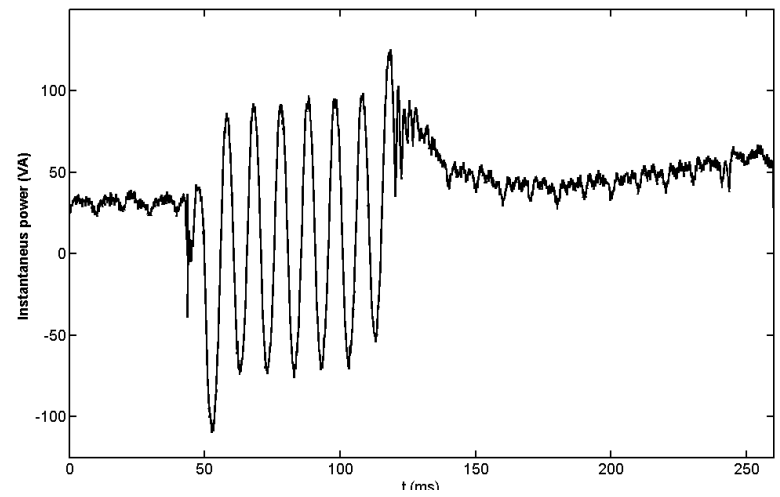

Fig. 11. Instantaneous power of the register

Figure 12 shows effective power, equation 2 and the reactive power, equation 3 , compared to the time evolution of the apparent power at the measurement point. It may be seen that at the time the fault occurs there is a peak of effective power that becomes negative.
This is because generation capacity on the LV side. This also explains why the voltages of the other two phases show large increases during the dip.

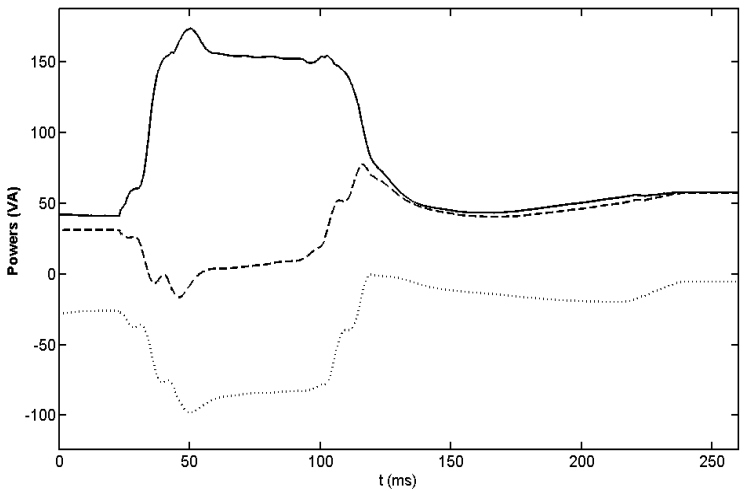

Fig. 12. Time evolution of the power expressions. Legend: - P. apparent. ---- P. effective. -.-.-. P. reactive.

The reactive power is negative, which means that the reactive capacitors are stuck, in the installation. The reactive power associated with the phenomenon of phase shift between voltages and currents of the same phase is much reduced during the dip. From this it may be deduced that the impedance of the fault is highly capacitive.

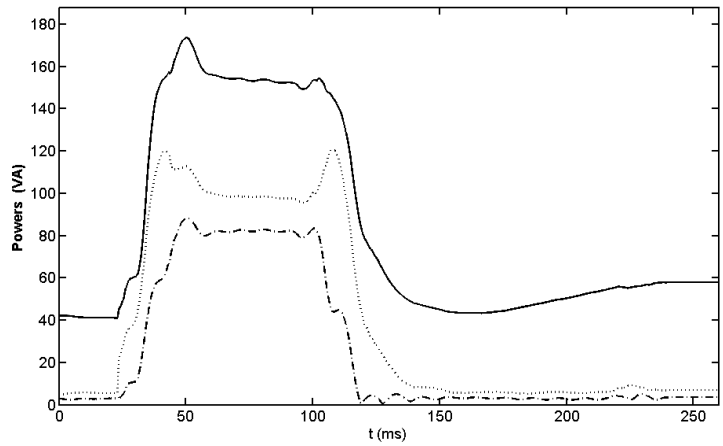

Fig. 13. Time evolution of power expressions Legend: -P. apparent. ---- P. distortion -.-.-. P. asymmetric.

Figure 13 shows how the asymmetric and the distortion power expressions increase during the fault. The distortion is maximised at the beginning and end of the fault, as those are the time when the signals are the most deformed. The asymmetry increases much during the dip. For a balanced system, the power is practically nil before and after the dip.

Lastly, the evolution of the efficiency during the fault is shown in figure 14. This efficiency is calculated according to equation 7 .

It may be observed that due to the large increase in absolute value of the reactive, distortion and asymmetric power expressions, the efficiency is practically reduced to zero. 


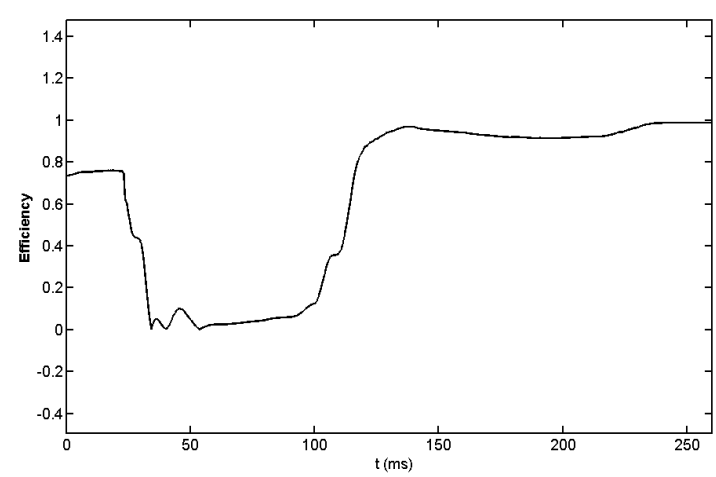

Fig. 14. Efficiency during the fault

\section{Conclusions}

This work has served to highlight the following points:

1) The STFT does not offer as much timefrequency resolution as might be desired. For this reason, it is necessary to arrive at a compromise between the detection of the perturbation and the exact measurement of the harmonics of the signal. Even so, the STFT affords much information about the perturbation.

2) The WT is better adapted to the detection of the beginning and end of the perturbation, but the STFT is the best method for the analysis of the harmonics.

3) The organization of the registers in database and their integration by means of MATLAB provides very good flexibility for the processing and representation of electrical data.

4) The current waveform provides useful information about the perturbations, and is also of interest from the charge standpoint.

5) The time evolution of the R.M.S. values of the voltages and currents may shed light on the physical mechanism of the perturbation.

6) The time evolution of the harmonics enables the detection of problems such as resonances or harmonics appearing after the perturbation. (e.g. dip caused by the starting of a faulty motor)

7) Theories of electrical power in non-sinusoidal regimes must be applied in order to analyse the perturbations from an energy standpoint.
8) The study of the energy of perturbations affords a broad view of the system, including all the voltage and current signal information.

9) The asymmetric power expression is good quantitative measure of the imbalance of the system. The distortion power expression is a good indicator of the deformation of the waves of the system.

10) The efficiency during the perturbation is a good indicator of the severity of the perturbation and includes all the effects which may be measured electrically.

\section{References}

[1] Bollen, M. H. J. and Irene Y.H.G., "Signal processing of power quality disturbances", IEEE Press, 2006.

[2] Mallat, S.G., "A Wavelet Tour of Signal Processing", Academic, 1999.

[3] F. Cavallé, C. Bonfil, and J. Martínez-Canales, "Studies of perturbations: their causes and effects on MT networks," Electricity Distribution, 1993. CIRED, 12th International Conference on, pp. 2 3,1993.

[4] C. Budeanu, "Reactive and Fictitious Powers," Inst. Romain de I'Energie, Bucharest, Rumania, 1927.

[5] S. Fryze, "Wirk-, Blind-und Scheinleistung in elektrischen Stromkreisen mit nichtsinusförmigem Verlauf von Strom und Spannung," Elektrotechnische Zeitschrift, vol. 25, pp. 569-599, 1932.

[6] N. Kuster and W. Moore, "On the Definition of Reactive Power under Non-Sinusoidal Condition." IEEE Trans. on Power Apparatus and Systems, PAS-99, pp. 1845-1854, 1980.

[7] Czarnecki, LS and Swietlicki, T., Powers in nonsinusoidal networks: their interpretation, analysis, and measurement, Instrumentation and Measurement, IEEE Transactions on, vol. 39, $\mathrm{n}^{\mathbf{0}} 2$, pp 340-345, 1990.

[8] L. Czarnecki, "New power theory of the 3-phase non-linear asymmetrical circuitssupplied from nonsinusoidal voltage sources," Circuits and Systems, 1988., IEEE International Symposium on, pp. 1627-1630, 1988.

[9] León, V., Método integral para la mejora de la eficiencia y la calidad en el suministro de la energía eléctrica en baja tensión., $\mathrm{Ph}$ Thesis, Universidad Politécnica de Valencia, September 1997.

[10] Montañana, J., "Medida integral de la eficiencia en el suministro de energía eléctrica en baja tensión. $\mathrm{Ph}$ Thesis, Universidad Politécnica de Valencia, 1999. 